\title{
La antropología en la investigación demográfica
}

\section{Susana Lerner}

Como parte de la investigación sociodemográfica, en especial de la realizada en América Latina, existe una tradición, al menos desde la década de los setenta, que se ha orientado al cuestionamiento continuo acerca de los diversos enfoques y estrategias teóricometodológicos para abordar el estudio de los fenómenos poblacionales y que remiten a la manera de aproximarnos a aprehender y comprender dichos fenómenos.

Lo anterior, en gran medida, obedece a la insatisfacción que se deriva de las limitaciones y alcances propios del quehacer demográfico clásico, donde se distinguen dos perspectivas independientes y a su vez complementarias. Por una parte, el análisis demográfico cuya orientación eminentemente cuantitativa da cuenta de los cambios en las tendencias y niveles de los fenómenos que caracterizan la dinámica demográfica, tales como la fecundidad, la nupcialidad, la mortalidad y las migraciones, así como las características asociadas a éstos que aluden a las diferencias que se observan en el nivel de individuos, familias, grupos sociales y ámbitos geográficos distintos. Entre éstas destacan los atributos relacionados con la edad, el sexo, los niveles de educación, categorías maritales y ocupacionales, entre otros. Bajo este acercamiento, y ante la delimitación teórica-metodológica inherente a esta disciplina, centrada en el quantum de dichos fenómenos, subyace una abstracción de la especificidad y significado social e histórico de éstos y de las características individuales asociadas a ellos.

Por otra parte, los estudios de población se identifican por buscar una comprensión y explicación de los fenómenos demográficos a la luz de la consideración de las dimensiones de los procesos sociales, económicos, culturales y políticos de la realidad social que inciden en dichos fenómenos y son a su vez influidos por ellos. No obstante, bajo esta perspectiva la investigación sociodemográfica se ha caracterizado, hasta mediados de la década de los ochenta, por privilegiar como parte de sus marcos explicativos los correspondientes a la sociología y la economía, enfatizando las determinaciones macro y microestructurales de las condiciones materiales de vida y las derivadas de situaciones de desigualdad social. Cabe agregar que las dimensiones culturales y subjetivas presentes en estas investigaciones, como por ejemplo las evidencias de las encuestas que se refieren a actitudes, ideales 
y motivaciones íntimas han sido analizadas con métodos cuantitativos sofisticados, o sea, bajo la óptica y tratamiento tradicional, en tanto variables independientes que reflejan atributos personales objetivados carentes de su significado cultural y subjetivo específico.

A pesar de los avances logrados como parte de esta última perspectiva, también subsiste una insatisfacción y cuestionamiento acerca de la utilización de los diversos marcos explicativos y metodológicos provenientes de otras disciplinas y, en especial, de sus resultados en términos de una aproximación y comprensión más integral y completa que permita dar cuenta de la complejidad de los comportamientos poblacionales, de sus determinantes, de la lógica o sentido social de sus acciones, de la multiplicidad de intermediaciones que en ellos intervienen y de sus consecuencias.

Por lo mismo, no es de extrañar que una parte importante de la reflexión y de la propia investigación sociodemográfica se haya orientado a la discusión de las cuestiones metodológicas inherentes a este campo, enfatizando la preocupación y necesidad de incorporar, combinar e integrar diversas dimensiones de la realidad, diferentes perspectivas disciplinarias y varios estilos y niveles de análisis para aproximarse a entender las relaciones entre el individuo y su entorno social y, así, profundizar en el significado que los individuos otorgan a sus propias acciones. Más aún, y como parte de la estrategia de investigación que permita estudiar la permanencia o los cambios relacionados con los fenómenos demográficos, el énfasis actual reside en dar cuenta de las percepciones, actitudes, valores, expectativas y prácticas como construcciones sociales, culturales y subjetivas inherentes al comportamiento de la población.

A esto último, y sin duda de manera simplista y poco rigurosa por parte de los estudiosos de las cuestiones de población, se le ha denominado perspectiva antropológica en la investigación sociodemográfica. Al respecto, existen numerosas evidencias acerca del interés creciente en la incorporación de dimensiones de y entre las disciplinas de la antropología y la demografía. Los estudios, sobre todo, en fecundidad y migraciones y más recientementemente los relacionados con los procesos de salud y mortalidad, con la sexualidad, con el papel de la mujer, de la familia y de las instituciones sociales en el comportamiento reproductivo, entre otros, dan cuenta de la diversidad de acercamientos analíticos bajo los cuales se busca incorporar el enfoque antropológico. En términos operativos lo anterior se ha traducido, de manera casi exclusiva, en investigaciones a nivel micro, o sea, en estudios de casos o en determinados contextos y en la utilización comple- 
mentaría o no de diferentes metodologías y técnicas, como, por ejemplo, las de tipo cualitativo, en especial las entrevistas en profundidad dirigidas a agentes y actores clave, el análisis de contenido de los discursos, las historias de vida y los grupos focales. También por el lado de los estudios antropológicos se observa un creciente interés por tomar en cuenta las teorías, los elementos y las herramientas del análisis demográfico. ${ }^{1}$

A su vez, como testimonio del interés creciente por la búsqueda de aproximaciones antropológicas en los estudios de población están las numerosas actividades y reuniones científicas de carácter nacional e internacional, tales como las realizadas por el Comité de Antropología y Demografía de la Unión Internacional para el Estudio Científico de la Población, ${ }^{2}$ por el Grupo de Trabajo con el mismo nombre de la Sociedad Mexicana de Demografía, ${ }^{3}$ las numerosas sesiones de diversos congresos que incluyen como temática las relaciones entre la demografía y la antropología ${ }^{4}$ y los cada vez más frecuentes talleres, cursos y seminarios sobre metodologías cualitativas en la investigación sociodemográfica. A ello se agregan las amplias referencias bibliográficas que dan cuenta de la relevancia que ha venido adquiriendo la actividad de investigación en esta línea. ${ }^{5}$

Los artículos reunidos en esta publicación, la mayoría de los cuales fueron presentados en una versión preliminar en la sesión de trabajo titulada "La antropología en la investigación sociodemográfica” en ocasión del XIII Congreso Internacional de Ciencias An-

1 A manera de ejemplo, podemos citar entre otros, los trabajos de Lourdes Arizpe, Óscar Cuéllar, Soledad González, Eduardo Menéndez, Gail Mummert.

2 Establecido en 1986 con ese nombre, aunque con anterioridad existía otro Comité denominado "Enfoque micro en la investigación demográfica".

3 Grupo de Trabajo establecido en 1992, cuya primera reunión de trabajo se realizó en enero de 1994.

4 Véase entre otros los diversos congresos generales, conferencias y seminarios organizados por la Unión Internacional para el Estudio Científico de la Población; las conferencias y reuniones latinoamericanas de población organizadas por diversas instituciones y programas de la región, tales como El Colegio de México, el Centro Latinoamericano de Demografía, el Consejo Latinoamericano de Ciencias Sociales, el Programa Latinoamericano de Actividades en Población; así como diversos eventos internacionales organizados por las Asociaciones Internacionales de Sociología y Antropología y las asociaciones nacionales de población como son la de Estados Unidos y Brasil. En el ámbito nacional, un ejemplo serían las reuniones nacionales organizadas por la Sociedad Mexicana de Demografía a partir de 1977.

5 Si bien no se cuenta con una sistematización bibliográfica sobre las investigaciones realizadas bajo esta perspectiva, las publicaciones de los diferentes eventos señalados en las notas anteriores son testimonio de la importancia creciente de la misma. 
tropológicas y Etnológicas (CICAE) que se llevó a cabo en la ciudad de México en agosto de 1993, representan un momento adicional de la reflexión en este intento de convergencia entre dos disciplinas. Dada la actualidad e importancia de las cuestiones tratadas en ellos, resulta relevante difundir en un público más amplio el conjunto de artículos de este congreso.

La realización de este evento respondió a la invitación del Comité Científico del CICAE para organizar, por parte del Centro de Estudios Demográficos y de Desarrollo Urbano, una sesión sobre las relaciones entre antropología y demografía, iniciativa que estuvo a cargo de la que suscribe esta presentación. La idea inicial consistió en reunir aportaciones de investigadores latinoamericanos que dieran cuenta de cómo diversos especialistas en temas de población visualizan y han incorporado la perspectiva antropológica en los estudios sociodemográficos.

La presente introducción sintetiza los principales hallazgos y recomendaciones de los artículos incluidos. Como el lector observará, se trata de un conjunto de contribuciones de naturaleza temática y de acercamientos analíticos muy diversos que tienen en común ofrecer una gama de reflexiones de carácter teórico-metodológico sobre la relevancia de incorporar la perspectiva antropológica en la investigación sociodemográfica y, algunos, mostrar los hallazgos empíricos resultantes que forman parte de experiencias concretas de investigación. En ellos, por lo mismo, se documenta una diversidad de problemas específicos, algunos de carácter propositivo y otros que representan exploraciones metodológicas pioneras, o reflexiones sobre el estado de conocimiento en una determinada temática y, en lo que respecta a los resultados empíricos, se refieren a grupos, poblaciones, contextos sociales y culturales particulares y heterógeneos que corresponden al universo de estudio.

Un primer grupo de trabajos consiste en propuestas conceptuales y metodológicas complejas que tienen como objetivo mostrar abordajes interdisciplinarios en la manera de analizar los procesos de población. Por la naturaleza de su contenido, estos artículos son los que aportan reflexiones de mayor alcance teóricoconceptual, constituyendo aportes relevantes e innovadores en la búsqueda de acercamientos alternativos y complementarios en la investigación sociodemográfica.

El artículo de Guarací Adeodato Alves de Souza contiene una compleja propuesta conceptual y metodológica. Partiendo del reconocimiento y consenso que existe entre los científicos sociales con respecto al avance cuantitativo y cualitativo en la investigación social e histórica en general, en que la antropología tuvo papel desta- 
cado, la autora considera que tales aportes resaltan la importancia de los sistemas simbólicos involucrados en distintas instancias y dimensiones de la vida social. Sin embargo, si bien tales aportes influyeron en los estudios de demografía social, al diversificar los temas, enfoques y métodos de investigación, no se lograron iguales avances en el tratamiento teórico-metodológico, ni en la claridad y consistencia de interpretación de las diferentes manifestaciones de los procesos demográficos o poblacionales, debido a las dificultades de diálogo entre los demógrafos formales y otros científicos sociales. Éstas son dificultades de "intercambios simbólicos" entre miembros de culturas científicas distintas, con lenguajes propios.

En el campo propio de la demografía, la autora menciona las limitaciones de la demografía formal, perspectiva que al aislar los fenómenos en un plano ahistórico, abstrae, por definición, todas las relaciones sociales y las dimensiones subjetivas y simbólicas que les garantizan existencia histórica. Por otra parte, en la tradición de la demografía social, que busca interpretar los fenómenos demográficos a partir de referencias analíticas producidas en otras disciplinas sociales, aún subsiste una explicitación poco clara e integrada de su naturaleza social, de la ubicación de la fecundidad, la mortalidad y el crecimiento vegetativo en las estructuras sociales, así como la forma en que los sujetos sociales los concretan en la vida social.

Compartiendo esta opinión, de la simplificación, ahistoricidad y abstracción de las teorías de la demografía formal, Guarací reconoce empero, que varios de los modelos teórico-conceptuales de la demografía formal constituyen los fundamentos de una problemática, la de la sucesión de las generaciones en cuanto proceso demográfico "puro", y por ello no pueden ser ignorados o sustituidos por otros enfoques. Con base en estas consideraciones reafirma la necesidad y posibilidad de un trabajo analítico, coherente con la visión disciplinaria de la demografía formal, de rescate de una perspectiva histórica de los procesos sociodemográficos. Para ello, parte del concepto de la sucesión de las generaciones, que en la teoría de la demografía formal es el proceso central de la dinámica demográfica global.

Como parte de la teoría de las poblaciones estables, la autora plantea que si bien los modelos desarrollados por Lotka se caracterizan por una elevada abstracción, con formulaciones de la fecundidad y la mortalidad como acontecimientos colectivos automáticos, sin autor ni protagonistas, éstos no presentan el sesgo biologicista que algunos demógrafos le atribuyen, sino que sus limitaciones mayores se hallarían en el tratamiento ahistórico, estrictamente cuantitativo y mecánico del proceso. 
En consecuencia, propone completar esta perspectiva incorporando componentes no demográficos, en cuanto mediaciones directas y decisivas, indispensables para la realización histórica y viabilidad de la reproducción demográfica. Lo anterior implica considerar los aportes sociológicos y antropológicos, como son las proposiciones sobre la familia como instancia de mediación para la determinación de la fecundidad y para el análisis directo de los momentos centrales de producción de las nuevas generaciones.

Con tales criterios, la autora explicíta que la sucesión de las generaciones, desde una perspectiva histórica, es un proceso constituido por el encadenamiento de diferentes tipos de acciones y relaciones sociales específicas, a saber: 1) el casamiento o el ejercicio de la sexualidad; 2 ) la procreación o reproducción de hijos nacidos vivos; 3) la preservación de la sobrevivencia y de la salud, y 4) la crianza-socialización de los hijos. Estos aspectos, son mediaciones directas y decisivas que operan en los planos material y simbólico, confieren especificidad a la reproducción humana, influyen en la propia subjetividad de los distintos actores involucrados y tienen, por lo tanto, un papel fundamental en la conformación de los patrones demográficos.

Para su continuidad histórica, estos patrones requieren cierta rutina y permanencia en el tiempo de algunos modos de articulación. De ahí que todas las sociedades establezcan normas, principios y reglas orientados a la conformación de determinados patrones de conducta y mecanismos de control social para la preservación de los mismos. La antropología ha mostrado que los códigos de parentesco y de matrimonio establecen vínculos simbólicos entre actores sociales con estatus distintos, atribuyendo valores y significados especiales a los papeles de los miembros de la familia. De este modo, los códigos de parentesco representan coordenadas culturales que sitúan a los individuos en la estructura de las relaciones sociales, confiriéndoles una posición que implica ciertas expectativas de conductas. Tales aportaciones de la antropología son decisivas para pensar en las dimensiones demográficas de la vida social como cursos de acciones impregnadas de elementos simbólicos y subjetivos. Por tanto, Guarací plantea que un patrón o régimen de sucesión determinado de las generaciones puede ser descrito en términos del modo en que los actores sociales vivencian (por la realización de ciertas prácticas y estrategias sociales y sus implicaciones directas) los distintos momentos de producción de sus descendencias, y un patrón determinado de procreación sería la realización histórica de un sistema de prácticas y estrategias de procreación en determinadas circunstancias materiales de vida, 
generando determinados resultados directos, en términos de las proles constituidas.

Ante la multiplicidad y diversidad de factores que pueden intervenir y afectar la continuidad de la realización histórica de un patrón de procreación, la autora señala la inutilidad de intentar definir a priori los que serían más decisivos -si los económicos, los demográficos, los ideológicos, los culturales o los políticos-, ya que, en general, todos estos factores interfieren en el cuadro de circunstancias favorables o desfavorables a la determinación de cierto patrón. En conclusión, la autora subraya la necesidad de continuar analizando la diferenciación, persistencia y cambio en los patrones de procreación como una de las cuestiones científicamente relevantes que precisan ser mejor estudiadas, con el apoyo de proposiciones analíticas más consistentes que las implícitas en los análisis de los "diferenciales de fecundidad" o en la teoría de la transición demográfica. El pensamiento científico en la demografía necesita ir mucho más allá de las cantidades y clasificaciones dicotómicas.

La relevancia de este artículo se inscribe en el continuado interés por contribuir a la construcción de una propuesta conceptual y metodológica que contemple las aportaciones de otras disciplinas así como las que se encuentran en los presupuestos de la demografía formal.

Como parte de los esfuerzos de la investigación sociodemográfica por desarrollar abordajes multidisciplinarios, el trabajo de Carolina Martínez constituye una propuesta metodológica de complementariedad de las perspectivas sociodemográfica, psicoantropológica y sociopsicoanalítica para profundizar en la comprensión y significado de las raíces individuales y familiares del comportamiento poblacional. Su trabajo parte de una excelente síntesis que nos recuerda los principales cuestionamientos de orden metodológico en la investigación sociodemográfica, que estaban presentes en el marco de los debates latinoamericanos en las décadas de los setenta y principios de los ochenta y que son retomados y desarrollados con gran precisión por la autora como antecedentes y elementos de la propuesta de las perspectivas psicoantropológicas y sociopsicoanalíticas que presenta.

La vertiente sociodemográfica trabaja fundamentalmente a partir de censos y encuestas cerradas y permite visualizar las relaciones que los fenómenos demográficos guardan con las variables económicas y sociales, ya sea en el nivel macro o micro, pero las explicaciones del comportamiento demográfico sólo llegan hasta un cierto nivel. El límite en el câso de las encuestas sociodemográficas, se encuentra en su incapacidad de dar cuenta de la tem- 
poralidad de los distintos procesos sociales, de los vínculos ideológicos o afectivos que las respuestas expresan veladamente y de las diferencias internas de los significados sociales y culturales.

La vertiente psicoantropológica, a partir de la aproximación microsocial y de entrevistas en profundidad, permite al investigador recuperar el manejo consciente, racional, creativo y afectivo como punto medular para la comprensión del comportamiento humano. Al respecto, la autora destaca la problemática que enfrenta el investigador en las interpretaciones y significados que le otorga a los distintos universos del discurso, a las ambiguas, contradictorias, congruentes o incongruentes respuestas y a los mensajes ocultos o aparentes de los sujetos entrevistados.

Sin duda, ello representa uno de los aspectos centrales en las investigaciones que tienen como objetivo indagar acerca del sentido de las percepciones, actitudes, normas, valores y expectativas que no son ajenas a la lógica subyacente en los comportamientos y acciones de los individuos. Lo anterior remite, en la terminología psicoanalítica, al manejo de la contratransferencia, o sea la transacción que opera entre el investigador y el sujeto estudiado y alude a la interacción y la reacción recíproca entre el observador y el observado, así como a la capacidad de reconstrucción objetiva del investigador frente al contenido y significado del material de investigación que los sujetos entrevistados le proporcionan.

A las proposiciones anteriores y como parte de la perspectiva sociopsicoanalítica o relacional, la autora agrega la incorporación de las dimensiones intrapsíquicas de los individuos y grupos para el abordaje de problemas de población. Con ello, rescata una de las cuestiones centrales de la investigación sociodemográfica que se refiere a la vinculación entre el individuo y la sociedad, en la cual se estructuran las relaciones interpersonales y la dimensión personal o intrapsíquica de cada individuo como parte del grupo social. Estas reflexiones reiteran la vital importancia otorgada al papel de las mediaciones en la investigación sociodemográfica, donde se ha destacado de manera especial el papel central de determinadas instituciones sociales como la familia o las instituciones y agentes de salud para dar cuenta de los comportamientos de determinados fenómenos poblacionales (varios de los artículos presentados en este volumen también retoman estos elementos).

Asimismo, en esta línea, los planteamientos de Carolina que enfatizan las dimensiones culturales de la naturaleza y condición humana, la determinación de lo que llama "carácter social", se asemejan a los ya mencionados por otros autores que subrayan la relevancia de considerar "la matriz de opciones" (Przeworski, 
1982)6 o el habitus (Bourdieu, 1979)7 a que se enfrenta o adquiere el individuo, y en donde las estructuras caracterológicas de los individuos que tienen su propia inercia se ven moldeadas por el efecto de las temporalidades propias y diferentes de los procesos de cambio socioeconómico y cultural. En este tipo de argumentación, de singular importancia, reside la posibilidad de expresar una distinción entre comportamientos heterógeneos, incluso en contextos supuestamente homogéneos (véase al respecto el artículo de Samuel et al. en esta publicación).

Como resultado de esta combinación de perspectivas y acercamientos, su sugerencia de desarrollar como estrategia analítica un "cuestionario interpretativo" resulta una aportación innovadora que permite, a diferencia de las investigaciones clásicas que privilegian una aproximación sociodemográfica y que conllevan a analizar las respuestas a cada pregunta desvinculadas del individuo que las proporcionó y de su propio contexto de socialización, examinar el conjunto de las respuestas para cada sujeto, rescatando así las particularidades propias del individuo en función de los diferentes ámbitos con los que se relaciona en las diversas etapas de su historia de vida.

Resulta evidente que en el trabajo de esta autora encontramos una reflexión metodológica que, a pesar de su carácter propositivo, revela no sólo la complejidad y pertinencia de la misma, sino la indudable relevancia de continuar realizando esfuerzos bajo una perspectiva psicosocial y antropológica diferente y a su vez complementaria a la que encontramos en la investigación sociodemográfica tradicional.

De manera similiar, Olivia Samuel, Susana Lerner y André Quesnel, proponen complementar la perspectiva demográfica de la nupcialidad con un acercamiento antropológico para analizar, en contextos rurales de México, los cambios experimentados en la naturaleza y significado de los procesos de conformación de la pareja y de la familia, la influencia de las relaciones que establecen los cónyuges entre sí y su vinculación con las acciones institucionales, en especial con las de salud.

Los autores dan cuenta de los aportes y las limitaciones del abordaje demográfico en el estudio de la nupcialidad y, sobre todo, de su vinculación con los cambios observados en los com-

6 Véase Adam Przeworski, “Teoría Sociológica y el estudio de la población: reflexiones sobre el trabajo de la Comisión de Población y Desarrollo de cLAcso", Reflexiones Teórico-Metodológicas sobre Investigaciones en Población, México, El Colegio de México, 1982.

7 Véase P. Bourdieu, "Les stratégies matrimoniales dans le systeme de reproduction", Annales E.S.C., núms. 4 y 5, pp. 1105-1125. 
portamientos reproductivos. Al respecto, cuestionan el hecho de la aparente estabilidad que caracteriza a la nupcialidad mexicana como resultado del alcance de los indicadores demográficos clásicos para su análisis y sugieren la necesidad de examinar sus posibles cambios a partir de la consideración de otras dimensiones que expresen la naturaleza y significado de este fenómeno y que sean susceptibles de ser incorporadas y traducidas en indicadores operativos a ser considerados como parte del análisis demográfico. Unido a ello, muestran como parte de este abordaje -ya sea que la nupcialidad sea considerada como objeto de estudio en sí mismo, como variable intermedia o dependiente de la fecundidad o bien en los estudios que vinculan los cambios de la fecundidad con los observados en el contexto social, económico y sanitario- la ausencia de toda consideración de los procesos sociales y culturales que inciden sobre la nupcialidad, y más aún la interrelación de ésta con el comportamiento reproductivo.

Por el lado de la antropología, la nupcialidad y en general los fenómenos demográficos no han sido objeto de estudio por sí mismos. Si bien éstos se conciben teóricamente como elementos constitutivos del sistema social, que repercuten y a su vez se ven influidos por dicho sistema, la investigación bajo esta perspectiva adolece de un reduccionismo al no tomar en consideración los cambios en las expresiones estructurales objetivamente construidas, al aislar y cosificar la lógica social propia del contexto y de los actores sociales considerados y al omitir o incorporar de manera marginal los efectos restructurantes de la dinámica demográfica.

En virtud de lo anterior, la propuesta de los autores combina un acercamiento de tipo antropológico con uno de índole demográfico. Con ello, se busca superar las limitaciones de cada uno de los abordajes considerados aisladamente e intentar integrar aproximaciones diferentes que den cuenta de las dimensiones subjetivas y de las relaciones interpersonales y con diversos ámbitos sociales, que se traducen en la adopción de prácticas e ideales que se inscriben en un proceso de cambio demográfico y cultural.

Las inquietudes expresadas por los autores acerca de la interdisciplinariedad y de la búsqueda de diferentes aproximaciones tiene como sustento varias premisas, que no están alejadas de los planteamientos de Carolina Martínez. Entre éstas destacan: la heterogeneidad del comportamiento de los individuos incluso en contextos supuestamente homogéneos y, aunado a ello, la distinción que opera entre individuos, parejas, familias y grupos sociales en donde intervienen dimensiones individuales que esconden procesos mucho más complejos que las simples características personales. Se trata de procesos que son reflejo de un capital so- 
cial, cultural, económico y religioso que adquiere el individuo en su proceso de socialización y que se va modificando en el transcurso de su trayectoria de vida individual y social. Dicho capital social influye no sólo en las percepciones, actitudes y valores particulares frente a la conyugalidad y la procreación, sino, también, en la modalidad de relaciones que los individuos establecen entre sí, con otros ámbitos y actores, determinando sus comportamientos, como serían la formación de la pareja y la procreación de la descendencia.

Por lo tanto, las proposiciones de los autores llevan a identificar un esquema de análisis que pone de manifiesto la interdependencia entre la nupcialidad y el comportamiento reproductivo. Como parte del mismo, se muestra la manera en que las modalidades y ritmos de adaptación de las parejas frente a las transformaciones sociales y a los cambios en los esquemas institucionales de procreación dependerán, en gran medida, de las formas y naturaleza de las uniones entre los miembros de ellas y, a su vez, repercutirán sobre la posición de ambos en las transacciones conyugales que se establecen entre la pareja y con el exterior.

La estrategia analítica utilizada ilustra el manejo de datos de naturaleza mixta, o sea la combinación de metodologías cuantitativas y cualitativas, así como la retroalimentación y complementariedad entre los enfoques demográfico y antropológico; es decir, la importancia de un acercamiento demoantropológico mediante el uso de encuestas sociodemográficas ad hoc y la realización de entrevistas semiestructuradas y en profundidad a los miembros de la pareja. Con base en ello, los autores identifican y construyen tipologías e indicadores objetivos que expresan dimensiones subjetivas y realizan un análisis de los discursos que arroja luz acerca del significado de la duración de la unión, los motivos de la misma y de la elección del cónyuge y las dimensiones afectivas presentes en dicha relación.

Entre los principales resultados alcanzados se muestra la relación entre la duración del noviazgo y la presencia o ausencia de proyectos de vida conyugales o familiares como expectativas en dicha etapa, y las diversas y no excluyentes opciones que subyacen en los motivos para la unión y, en particular para la selección del cónyuge. Entre éstas últimas destacan las de naturaleza utilitaria como reflejo de situación de carencias, conflicto y control autoritario y represivo en el ámbito familiar de origen, la presencia de elementos sentimentales que aluden a elementos afectivos y la que se desprende de la sumisión a la normatividad social como expresión de una construcción social débil de la individualidad frente a opciones de la vida. 
Vinculado con las dimensiones anteriores, el texto presenta algunos de los hallazgos que evidencian la estrecha vinculación e influencia entre la naturaleza de las uniones, las transacciones conyugales y la presencia o no de consenso entre los miembros de la pareja en cuanto a la constitución inicial de su descendencia.

Además, en este marco de relaciones se enfatiza la singularidad y relevancia de elementos culturales y sociales que intervienen, tales como la ignorancia sobre el funcionamiento del cuerpo, los tabués existentes sobre la sexualidad, las condiciones de desigualdad entre géneros, la persistencia de valoraciones normativas en la identidad femenina, entre otros.

Unido a lo anterior y frente a los cambios que se observan en la posición de la mujer y del hombre como resultado de las acciones de las instituciones de salud en materia de planificación familiar y de la transacción de éstas con los actores, se evidencia una mayor autonomización relativa de la mujer frente a las opciones reproductivas de su pareja, proceso que se ve acompañado por la mayor responsabilidad otorgada a ella en este proceso y una marginación progresiva del hombre como resultado de las opciones y responsabilidades de éste, ante la procreación y práctica reproductiva.

La exploración realizada por los autores constituye un trabajo pionero, que va más allá de una propuesta interdisciplinaria avanzando en la búsqueda que posibilite una integración de las dimensiones subjetivas y de las relaciones interpersonales en el análisis de los procesos poblacionales.

En su artículo, Juan Guillermo Figueroa reconstruye un proceso de investigación utilizado para el estudio de la esterilización femenina, mostrando los diferentes marcos conceptuales, enfoques metodológicos, temáticas desarrolladas y experiencias concretas en el conjunto de las sucesivas investigaciones realizadas en el ámbito de la Dirección de Planificación Familiar de la Secretaría de Salud.

Las contribuciones del artículo son tanto de tipo analítico como metodológico. En el primer plano, el autor apunta claramente a los interrogantes que surgen al observar la alta prevalencia de la esterilización femenina en México, su intensificación en un lapso de tiempo reducido y las propias características de este método, así como las de la población que ha optado por él. Interrogantes que lo llevan a enfatizar la necesidad de indagar acerca de los procesos y mecanismos que subyacen en el proceso de toma de decisiones por parte de la población con respecto a la opción por este método, a la reacción de ella en función de la experiencia vivida al respecto y, en especial, a cuestionar el papel y las 
acciones de las instituciones y agentes de salud en dicho proceso. Sin lugar a dudas, se trata de una problemática altamente relevante dado el papel protagónico de los prestadores de servicios de salud, que se inscribe como parte de la institucionalización medicalizada de las prácticas anticonceptivas en nuestro país y que se considera como uno de los determinantes fundamentales de los cambios en el comportamiento reproductivo de la población.

Las reflexiones del autor se inscriben, a su vez, en el debate actual acerca de los derechos reproductivos y las cuestiones éticas, que aluden a la responsabilidad de los distintos actores en la toma de decisiones para optar por este método, así como a los efectos de esta práctica anticonceptiva en la salud reproductiva e integral de las mujeres. Más aún, dicha práctica la ubica en el contexto mexicano caracterizado por amplias desigualdades en las relaciones de género, por situaciones de conflictos en la interacción entre la población y los agentes de salud, por las relaciones de poder y sumisión ante el saber médico, por las condiciones de acceso limitado a servicios y por la normatividad cultural vigente y cambiante en torno a la reproducción que no está exenta de incertidumbres, ambivalencias, contradicciones y que responde a múltiples y variadas valoraciones con respecto al significado de la reproducción, en particular en cuanto a la identidad femenina.

En el plano metodológico, las propuestas coinciden con las mencionadas en los otros artículos en cuanto a la necesidad de un acercamiento antropológico, psicosocial y complementario al sociodemográfico que permita una mejor aproximación para conocer las normas, valores y significados subyacentes en los procesos de reproducción humana, en particular en relación con los complejos procesos de toma de decisión anticonceptiva. Lo novedoso de este artículo es la estrategia seguida en dicha búsqueda, que consiste en un proceso de retroalimentación constante que incorpora el análisis de nuevas inquietudes derivadas del estado de conocimiento alcanzado en investigaciones ya realizadas y, con base en ello, la implementación de otros acercamientos. Así, una primera etapa parte de diferentes y nuevos análisis de la información recogida en las respuestas textuales de las encuestas de fecundidad realizadas en México (ENFES, EDEPAM), orientados a rescatar aspectos relacionados con derechos reproductivos, educación reproductiva y enfoque de género.

De acuerdo con las limitaciones e interrogantes no resueltos en estos instrumentos de recolección de información, una etapa adicional consistió en búsquedas específicas que dieran cuenta de las determinaciones culturales y de nuevas interpretaciones, con diferentes acercamientos que incluyeron problemáticas parti- 
culares y complementarias en torno a la multiplicidad de actores, instituciones, intereses, relaciones y dimensiones involucrados en los procesos de decisión sobre la reproducción y con especial énfasis en la práctica de la esterilización femenina. Las aproximaciones metodológicas adicionales de tipo cualitativo consistieron por una parte en entrevistas realizadas a los médicos, como prestadores de servicio de planificación familiar, con el objeto de conocer sus percepciones e interpretaciones en la relación entre la anticoncepción y los derechos reproductivos. Por la otra, se privilegió la utilización de historias de vida temática aplicadas a mujeres, incorporando elementos orientados a reconstruir parte del proceso de su identidad y su valoración como mujeres y madres y en su relación con la valoración de la anticoncepción quirúrgica.

El texto hace referencia a múltiples hallazgos y elementos críticos que se desprenden de los diferentes niveles de análisis y de los diversos ejes analíticos y acercamientos privilegiados. Entre estos destacan el cuestionamiento del autor acerca de la existencia de procesos diferenciales de valoración en los grupos poblacionales considerados, la importancia de la participación de las mujeres en el proceso de toma de decisiones en su contexto cotidiano, así como la presencia o ausencia de una percepción sobre sus propios derechos. Aunado a ello, y como temática novedosa e insuficientemente tratada en la investigación sociodemográfica, el análisis del papel de los prestadores de servicios, como agentes sociales del cambio demográfico y del comportamiento reproductivo de la población, para situar de mejor manera el contexto institucional y normativo en el cual se desarrolla el proceso de toma de decisiones de la población con respecto a sus prácticas reproductivas y anticonceptivas, así como la relación entre los derechos y responsabilidades de la población y de los prestadores de servicios.

Por su parte, Ivonne Szasz revisa la investigación que se ha llevado a cabo en México sobre migraciones femeninas y examina los aportes de los estudios sociodemográficos y antropológicos que consideran la condición de desigualdad de género como determinante de las modalidades y características que asumen los flujos migratorios y la inserción de las mujeres migrantes en los lugares de origen y destino. A estas condiciones de desigualdad agrega la necesidad de incorporar construcciones culturales que limitan las posibilidades de autonomía personal, de participación social y de movilidad de las mujeres.

Constatando el señalamiento que la literatura actual hace sobre las relaciones de género como determinantes de la especificidad de la movilidad espacial de las mujeres, la autora considera que la migración femenina requiere ser analizada como objeto de 
estudio autónomo, diferente de los desplazamientos masculinos, a partir de los factores que condicionan sus modalidades específicas, como son la división sexual del trabajo, el papel asignado a las mujeres en la reproducción y las diferencias de acceso a mercados y condiciones de trabajo, factores que determinan que el contexto familiar sea más importante en las migraciones femeninas que en las masculinas.

Ivonne da cuenta de la trayectoria seguida bajo la perspectiva sociodemográfica en los estudios de migración, especialmente los relacionados con la movilidad femenina, destacando las dimensiones relacionadas con la posición de la mujer y las relaciones sociales de género que se enfatizaron en distintos momentos de esta trayectoria. Así, los estudios más clásicos de la demografía en el nivel interestatal y en las principales metrópolis del país, mostraron la supremacía de la migracion femenina frente a la masculina en determinadas etapas y sus diferencias según contexto de origen, estado civil, inserción en el trabajo en el lugar de origen, así como según la selectividad por edad y nivel educativo.

Con posterioridad, los estudios realizados en contextos sociales específicos, y bajo una perspectiva interdisciplinaria que rescataba los aportes de la antropología, advertían acerca de los cambios y diversidad de los movimientos espaciales de las mujeres, mostrando la importancia de dichos movimientos a zonas urbanas de la frontera norte, el aumento de flujos femeninos hacia Estados Unidos y una mayor incidencia de las mujeres en movimientos temporales e itinerantes. En ellos y bajo el paradigma históricoestructural se destacaba la importancia de procesos más amplios como el de la reproducción demográfica y social en el análisis de la migración femenina. Este paradigma, utilizado tanto por la antropología como por la sociodemografía, analiza los migrantes no ya como individuos o familias aisladas, sino como actores insertos en clases sociales y rescatando a la familia como unidad preferente de análisis.

Ivonne considera que si bien los estudios antropológicos no se ocuparon específicamente de las relaciones de género, si permitieron superar el enfoque individualista, al rescatar el contexto comunitario y regional, la importancia del cambio agrario y del mercado matrimonial, y el uso de la unidad doméstica como unidad de análisis y los estudios longitudinales. Agrega que otra de las contribuciones antropológicas al conocimiento de las relaciones entre migración y género se refiere al impacto de la movilidad espacial y de la inserción laboral femenina en la condición de subordinación de las mujeres protagonistas.

Si bien su trabajo, comparado con los aquí publicados, difiere 
en la temática central que analiza, advierte, como parte de los hallazgos en el estudio de las migraciones femeninas, del efecto de socialización de los diferentes contextos de migración en la autonomía de las mujeres, en su capacidad de decisión y control sobre sus acciones y, por lo tanto, en comportamientos relacionados con la reproducción y anticoncepción.

Finalmente, la autora concluye su presentación señalan do nuevas interrogantes y necesidades de investigación que requieren un abordaje cualitativo e interdisciplinario del tema, partiendo del panorama general de las corrientes migratorias femeninas que las encuestas demográficas permiten configurar y de la necesaria complementación analítica con la visión antropológica, dados los determinantes culturales de las migraciones femeninas.

Un segundo bloque de artículos presenta resultados de investigación en que se empleó un abordaje antropológico para analizar dimensiones casi inexploradas y altamente relevantes que tienen un impacto sobre el comportamiento reproductivo y las prácticas anticonceptivas, como son las relacionadas con la sexualidad y con las percepciones, creencias y representaciones sobre el cuerpo femenino. A ello se agrega un trabajo que explora las relaciones entre los costos y beneficios económicos de los hijos como marco analítico, ampliamente debatido, para dar cuenta de los cambios en dicho comportamiento.

Carlos Aramburú y Rosario Arias dan cuenta de los resultados de una investigación comparativa sobre sexualidad y comportamiento reproductivo en mujeres de sectores populares del Perú. El marco conceptual de su estudio añade al modelo de transición demográfica uno de transición cultural, que desde la perspectiva antropológica privilegia el análisis de los cambios del rol y estatus de la mujer para poder interpretar las transformaciones con respecto a su sexualidad y vida reproductiva.

Sus reflexiones y propuestas son parte de las inquietudes expresadas en algunos de los otros artículos que se incluyen en la presente publicación, y en general en la investigación sociodemográfica actual, que centran su atención en la construcción cultural que posibilita dar sentido y enriquecer la comprensión de los resultados cuantitativos que se obtienen a través del uso de las herramientas e indicadores demográficos, en especial los que muestran diferenciales en el ritmo, nivel y características del comportamiento de la fecundidad. En el caso particular de este artículo, los autores incorporan las dimensiones culturales que están ausentes en el modelo de Bongaarts sobre los determinantes próximos de la fecundidad, centrando su análisis en las cuatro variables intermedias que son las que tienen mayor fuerza explicativa para analizar 
la variación entre los niveles de fecundidad: nupcialidad, lactancia, contracepción y aborto.

Como parte de la perspectiva antropológica, también, la estrategia de análisis que privilegian se apoya en la complementariedad de diversas técnicas cualitativas que tienen alcances diferentes. Por un lado, las encuestas con entrevistas en profundidad que incluyen historias de vida sobre diversas temáticas específicas para construir la experiencia individual en las etspas claves de la vida sexual y reproductiva de las mujeres y, por otra parte, los talleres grupales, como técnica de obtención de información para conocer las normas, valoraciones y apreciaciones colectivas en torno a distintas dimensiones de la sexualidad, y para confrontarlas con la experiencia individual recogida en las historias de vida. Mediante el uso de ambas técnicas los resultados exponen no sólo la presencia de una sexualidad femenina popular llena de contradicciones, temores y situaciones de subordinación, sino las contradicciones evidentes que afloran entre los valores grupales y la conducta individual en torno a la sexualidad.

No obstante las limitaciones que los propios autores señalan respecto de la metodología utilizada, entre las que destacan el alcance de la información para explicar la diversidad cultural y demográfica de la población, la mayor confiabilidad y amplitud de elementos a considerar en temáticas más complejas, la interacción entre el investigador y el investigado, así como la propia interpretación de los resultados por parte del primero, los resultados encontrados muestran la importancia de privilegiar y continuar en esta línea de análisis para una búsqueda de mejores explicaciones.

Como parte de la perspectiva sociocultural que busca aprehender y confrontar las normas y valores de los diferentes grupos de la población estudiada con la experiencia vivida por ella, su análisis se centra en dos niveles de análisis que los autores consideran como elementos explicativos fundamentales de la diversidad sexual y reproductiva de las mujeres estudiadas: la generación y el contexto cultural. De acuerdo con lo anterior, los autores reconocen la presencia de un proceso de homogeneización de la cultura que está acompañado de la socialización de la sexualidad entre las mujeres más jóvenes, frente a un contexto socializador más diferenciado e influido, en mayor medida, por el entorno familiar entre las generaciones más viejas. A la par, demuestran cómo la diversidad de comportamientos es resultado de la presencia de conflictos generacionales y de género, así como las divergencias entre el deber ser y las prácticas reales de cada uno de los grupos estudiados.

La riqueza y amplitud de las evidencias que presentan derivadas de los testimonios de las entrevistas en profundidad y de los 
talleres grupales, muestra la relevancia de la incorporación de dimensiones culturales para dar cuenta de la complejidad de la sexualidad temprana, así como para entender la naturaleza de la relación del noviazgo, la concepción prenupcial y las prácticas anticonceptivas, o sea el proceso de toma de decisiones y la conducta en torno al embarazo, la anticoncepción, y en general la sexualidad y comportamiento reproductivo. Entre los aspectos que subyacen en dichos comportamientos destacan la ausencia de información o desinformación por parte de los ámbitos familiar, escolar y por parte de las instituciones de salud, la presencia de prejuicios y tabúes respecto al uso de métodos anticonceptivos, la inexistencia o poca claridad de expectativas en la vida futura de los jóvenes, las condiciones de sumisión de las mujeres a su entorno familiar, institucional y social, el autoritarismo del varón y la ausencia de planeación y control por parte de la muijer, sumado a la permanencia de roles tradicionales genéricos.

Sin duda, la relevancia de este artículo es el abordaje de una temática casi inexplorada en los estudios sobre el comportamiento reproductivo, y que no sólo subraya la debilidad de la cultura anticonceptiva existente en la gran mayoría de los países de la región latinoamericana, a pesar del impacto de la anticoncepción moderna, sino, y más aún, de la inexistencia de una cultura sexual como motor de cambio, no sólo de dicho comportamiento, sino de las condiciones de salud reproductiva y en general de vida de las mujeres.

El trabajo de Elena Zúñiga y Daniel Hernández presenta un análisis de la importancia de los hijos para el mantenimiento de la población de la tercera edad, en tres comunidades rurales de México. El interés de esta problemática, que prácticamente no ha sido investigada en México, reside en su vinculación con las hipótesis relativas a la transición de la fecundidad. El problema de la percepción sobre los aportes y costos de los hijos se halla en el centro de esta elaboración teórica, sobre todo en la hipótesis que plantea que mientras los hijos signifiquen un valor económico para sus padres y la única seguridad en la vejez, la fecundidad se mantendrá elevada.

Si bien los autores no retoman un abordaje interdisciplinario, metodológicamente recurren a un uso combinado de técnicas sociodemográficas y antropológicas, ya que a la par de aplicar cuestionarios estructurados a todos los hogares con el fin de obtener datos sobre las características de las familias, la organización del trabajo familiar y la conducta reproductiva, recopilaron historias de vida de una submuestra de parejas o personas de 60 años o más, así como entrevistas a personal de salud y líderes comunitarios. 
A partir de la información recopilada y tomando en cuenta las características e historia de las comunidades consideradas, los autores presentan los resultados obtenidos utilizando dos conceptos base: la unidad doméstica y el ciclo familiar. De esta manera, proporcionan una visión en el tiempo del valor otorgado a los hijos en las diferentes etapas vividas por los miembros de la unidad doméstica, desde su conformación a partir de la unión de una nueva pareja y su independización del hogar paterno, hasta la llegada de los hijos, su crecimiento y finalmente, su salida del hogar paterno. Así, partiendo de la estructura y dinámica de las unidades domésticas y de los hogares de los viejos, los autores analizan el papel de los hijos a lo largo del ciclo familiar, las formas de manutención de las personas mayores y sus preferencias sobre el tamaño de la familia.

Como parte de esta problemática, es de gran interés el análisis del papel de los hijos en diferentes etapas del ciclo familiar. Los autores destacan que la percepción de los padres sobre el valor de los hijos tiene un fuerte contenido económico o de asistencia instrumental, pero que esta percepción va cambiando conforme los hijos van transcurriendo por su ciclo de vida individual. En la etapa de vida familiar caracterizada por la presencia de hijos pequeños, el peso reside en el costo de los mismos, frente a la siguiente etapa del ciclo familiar, cuando los niños tienen entre 8 y 14 años de edad, donde su aportación es percibida como marginal al participar éstos en las tareas agrícolas y domésticas. Finalmente, los mayores beneficios se alcanzan cuando los hijos tienen una mayor capacidad productiva y pueden optar por un trabajo asalariado, generalmente fnera de la localidad. Es en esta etapa cuando el monto de ingresos monetarios alcanza la máxima cantidad que los hijos brindan a sus padres, apoyo basado en la "obligación" de los hijos de ayudar a los padres, y que aparentemente no se sustenta más que en los lazos de solidaridad y en el altruismo de hijos a padres.

Es indudable que los hijos juegan un importante rol económico o de asistencia instrumental durante la vida; sin embargo, los autores destacan el hecho de que poco más de una quinta parte de las parejas o individuos de 60 años o más de su universo de estudio no reciben ayuda económica por parte de ningún hijo en la región, tratándose en general de las parejas o individuos más pobres. Los autores atribuyen esta desigualdad actual a un acceso diferenciado a los recursos productivos desde los inicios de las unidades de producción.

En conclusión, parecería que la explicación del cambio en los niveles de fecundidad que ha ocurrido en estas comunidades se ubica más en el nivel de los costos de la reproducción que en el de sus beneficios económicos. Los autores sugieren que en la ex- 
plicación de la reducción del número de hijos deben ser considerados aspectos relativos a la condición de género y pobreza y que es necesario ahondar en los mecanismos que debilitan los elementos de solidaridad familiar.

Por último, el documento de Ondina Fachel Leal consiste en una exploración etnográfica sobre creencias, representaciones y prácticas que afectan dimensiones del comportamiento reproductivo y la anticoncepción. ${ }^{8} \mathrm{~A}$ diferencia de los demás artículos, la autora no presenta una propuesta interdisciplinaria ni de complementariedad entre abordajes demográficos y antropológicos, sino que da cuenta de estas dimensiones con base en las evidencias de diversas investigaciones realizadas en grupos de mujeres en diversos contextos populares del sur de Brasil.

La problemática que desarrolla la autora se centra en el significado que adquieren las creencias y representaciones de las mujeres respecto a su cuerpo, para aproximarse a comprender la lógica cultural reproductiva y anticonceptiva a las que se enfrenta la población ante las opciones y acciones por parte de las instituciones de salud. La premisa fundamental que guía su trabajo es la visualización de los periodos menstruales y reproductivos de manera yuxtapuesta y altamente interrelacionada y, en vista de ello, su efecto directo sobre el uso de anticonceptivos. Para ello hace referencia a dos aspectos principales. El primero remite a comprender la lógica de tipo simbólico que ordena estas representaciones en relación con el cuerpo, sus líquidos y la concepción. El segundo, se refiere a las consecuencias de esta visualización en la selección y uso de anticonceptivos.

No obstante que se trata de un abordaje clásico de los estudios antropológicos, la autora intenta rescatar el sentido de las normas y valores culturales de determinados grupos de población, cuyas representaciones son retomados de manera errónea por parte del discurso y lógica biomédica que las conciben como parte de la cultura tradicional, retrasada e ignorante. A su vez, muestra como dicho discurso se entrelaza y es aprehendido por parte de la población como parte de una cosmovisión del mundo y de un sistema de representaciones sociales y culturales específico con respecto al cuerpo. Esta distancia entre el saber médico y el saber popular representa, sin duda, un elemento muy significativo que debe ser tomado en cuenta al considerar el mayor o menor éxito de la práctica anticonceptiva.

8 Dada la naturaleza exploratoria y preliminar de este trabajo que requiere un desarrollo más integral de las ideas expuestas, y la relevancia y novedad del tema tratado, el mismo se incluye en la sección de notas y comentarios de esta publicación. 
Las percepciones, creencias y reinterpretaciones simbólicas sobre el cuerpo femenino y los periodos de mayor riesgo, el significado y reinterpretación del embarazo y de la menstruación en relación con la fertilidad, el sentido mágico atribuido al uso de medicinas tradicionales y modernas, de prácticas alimenticias, de estados físicos y emocionales del cuerpo representan una búsqueda novedosa de explicaciones alternativas acerca del significado e impacto de las prácticas anticonceptivas y una dimensión ausente y altamente relevante en los estudios de población.

Finalmente, a modo de conclusión, es importante reiterar los motivos que guiaron la publicación de los trabajos presentados en este volumen de la Revista Estudios Demográficos y Urbanos. Por un lado, dar cuenta de un momento más de la relevancia del quehacer interdisciplinario, en este caso entre la demografía o la investigación sociodemográfica y la antropología, como campos disciplinarios con marcos analíticos y herramientas que son distintas, pero que constituyen alternativas complementarias para abordar el mismo objeto de estudio: las poblaciones humanas y sus transformaciones. Por otro lado, el mostrar la riqueza de continuar promoviendo espacios de intercambio entre profesionales de diversas disciplinas para identificar nuevas líneas y estrategias de investigación. En este sentido, la convergencia entre la demografía y la antropología, no sólo se sitúa en términos de la relación entre la investigación en el nivel micro y macro, entre la aprehensión de comportamientos particulares y procesos generales, o en el uso de metodologías cuantitativas y cualitativas, sino en la búsqueda de acercamientos alternativos que coadyuven en la reconstrucción de marcos interpretativos diferentes y complementarios para aproximarnos a una mejor comprensión del comportamiento de la población y, por ende, de una misma realidad.

Aunado a lo anterior, los resultados de esta acción interdisciplinaria ponen el acento en una de las omisiones centrales de la investigación sociodemográfica: la no consideración de las vivencias y experiencias (comportamientos) de los actores en cuanto sujetos de sus acciones y de las que le son impuestas y no sólo como objetos de estudio. En este sentido también debe subrayarse la importancia de la convergencia transdisciplinaria, en la medida que aporte elementos a ser considerados en la elaboración e implementación de programas y acciones que se llevan a cabo en materia de políticas de población y en general políticas sociales y en donde los individuos, las familias y los grupos sociales son únicamente considerados como sujetos pasivos. 
